Pacific

Journal of

Mathematics

ON THE NULLCONE OF REPRESENTATIONS OF REDUCTIVE GROUPS

HANSPETER KRAFT AND NOLAN R. WALlaCh

Volume $224 \quad$ No. 1

March 2006 


\title{
ON THE NULLCONE OF REPRESENTATIONS OF REDUCTIVE GROUPS
}

\author{
HANSPETER KRAFT AND NOLAN R. WALlaCH
}

\begin{abstract}
We study the geometry of the nullcone $N=N_{V^{\oplus k}}$ for several copies of a representation $V$ of a reductive group $G$ and its behavior for different $k$. We show that for large $k$ there is a certain "stability" with respect to the irreducible components. In the case of the so-called $\theta$-representations, this can be made more precise by using the combinatorics of the weight system as a subset of the root system. All this finally allows us to calculate explicitly and in detail a number of important examples, such as the cases of 3- and 4-qubits, which play a fundamental rôle in quantum computing.
\end{abstract}

\section{Introduction}

Let $V$ be a finite dimensional complex representation of a complex reductive group $G$ and denote by $N_{V} \subset V$ the nullcone, i.e., the zero set of the homogeneous nonconstant invariant functions on $V$. This cone was introduced and studied by Hilbert in his famous paper "Über die vollen Invariantensysteme" [1893], where he gives a "constructive" proof of the finiteness theorem for the invariants.

The nullcone $N_{V}$ plays a fundamental rôle in the geometry of the representation, in particular, in problems concerning the structure of orbits and their closure and in the study of the algebraic quotient $\pi_{V}: V \rightarrow V / / G$ given by the invariants. For example, if $f_{1}, f_{2}, \ldots, f_{r}$ are algebraically independent homogeneous functions defining the nullcone $N_{V}$, the invariants $\mathbb{C}[V]^{G}$ form a free module over the polynomial ring $\mathbb{C}\left[f_{1}, f_{2}, \ldots, f_{r}\right]$. Knowing the degrees of the $f_{i}$ 's one can immediately give an upper bound for the degrees of a generating system for the invariants. Moreover, there are efficient tools to calculate the Hilbert series of the invariants. We refer the reader to [Derksen and Kraft 1995] and to the book [Derksen and Kemper 2002] for more details.

In trying to understand the geometry of the $n$-qubits, i.e., the representation $Q_{n}:=\mathbb{C}^{2} \otimes \mathbb{C}^{2} \otimes \cdots \otimes \mathbb{C}^{2}$ ( $n$ factors) of $\mathrm{SL}_{2} \times \mathrm{SL}_{2} \times \cdots \times \mathrm{SL}_{2}$ (also $n$ factors, with

MSC2000: 20G20, 20G05, 14L24.

Keywords: nullcone, invariant, qubit, $\theta$-representation.

Kraft was partially supported by the SNF (Schweizerischer Nationalfonds), Wallace by an NSF summer grant. 
obvious action on $Q_{n}$ ) we became interested in the structure of the nullcone $N_{V^{\oplus k}}$ for $k$ copies of a given representation $V$ and in particular in its behavior for large $k$. It turned out that there is a "stability" property saying that the general structure does not change anymore once $k$ is greater than a certain number $m(V)<\operatorname{dim} V$ calculated from the weight system of $V$ (see Theorem 1.4 for a precise statement). For instance, for the 3-qubits $Q_{3}$ we find that the nullcones $N_{Q_{3}}$ and $N_{Q_{3}^{\oplus 2}}$ are both irreducible, of dimension 7 and 11 , respectively. The nullcone of $k \geq 3$ copies has 4 components, one of dimension $3+4 k$ which is stable under $S_{3}$ and three of dimension $1+4 k$ which are permuted by $S_{3}$ (see Example 2.4).

On the other hand, the nullcone of any number of copies of the adjoint representation of a semisimple group $G$ on its Lie algebra $\mathfrak{g}$ is irreducible and has a nice resolution of singularities, namely

$$
G \times{ }_{B} \mathfrak{n}^{\oplus k} \rightarrow N_{\mathfrak{g}^{k}}
$$

where $B \subset G$ is a Borel subgroup and $\mathfrak{n}:=$ Lie $B_{u}$ is the Lie algebra of the unipotent radical of $B$. A similar behavior can be found for all representations of $\mathrm{SL}_{2}$ where the nullcone for any number of copies is irreducible. But this behavior is rather exceptional for groups of rank $\geq 2$ and seems to occur only for "small" representations, such as the standard representation of $\mathrm{SL}_{n}$ (and its dual) and the 7-dimensional representation of $G_{2}$. We do not know a classification of these representations.

The next type of examples we study are the $\theta$-representations introduced by Vinberg [1976], motivated by and generalizing the situation of symmetric spaces studied by Kostant and Rallis [1971] (see Section 3). One of our reasons for this study is that the representation we called 4-qubits $Q_{4}$ is an example of a $\theta$ representation (see Section 4). In these cases we are able to prove more precise results (see Proposition 3.11 and Corollary 3.13), which enable us to determine the nullcone for many important examples. For instance, in the case of 4-qubits we find that $N_{Q_{4}}$ is irreducible, whereas for $k \geq 2$ the nullcone $N_{Q_{4}^{\oplus k}}$ has 12 irreducible components, decomposing into 3 orbits of 4 elements under $S_{4}$. The dimensions are $8 k+4,8 k+3$ and $8 k+1$ (Proposition 4.1). As already said, a key point of this paper consists in the examples worked out in detail, which show a very interesting behavior of the nullcone, mostly irregular and sometimes quite surprising. Although we do not have a complete picture or a final answer - maybe there is none - we believe that the general results explain some of the phenomena and that the examples will help to get a deeper insight into the situation.

Note. After finishing this paper we were informed about a paper of V. L. Popov [2003], where he gives a general algorithm to determine the irreducible components of maximal dimension of the nullcone, using the weights of the representation and their multiplicities. 


\section{Irreducible components of the nullcone}

Our base field is the field $\mathbb{C}$ of complex numbers. Let $G$ be a connected reductive group and $\rho: G \rightarrow \mathrm{GL}(V)$ a finite dimensional (rational) representation. The nullcone $N_{V}$ of the representations $V$ is defined by

$$
N_{V}:=\{v \in V \mid \overline{G v} \ni 0\}=\pi_{V}^{-1}\left(\pi_{V}(0)\right),
$$

where $\pi_{V}: V \rightarrow V / / G$ is the quotient morphism (see [Kraft 1984, Kap. II] or [Mumford et al. 1994]). The nullcone plays a fundamental rôle in the study of the geometry of the representation $V$ and of the quotient morphism.

In this section we will describe the irreducible components of $N_{V^{\oplus k}}$ for the representation of $G$ on $V^{\oplus k}=V \oplus V \oplus \cdots \oplus V$ ( $k$ copies of $\left.V\right)$ and show how $N_{V^{\oplus k}}$ behaves for $k \rightarrow \infty$.

By the Hilbert-Mumford criterion we know that a vector $v \in V$ belongs to the nullcone $N_{V}$ if and only if there is a one-parameter subgroup (abbreviated: 1-PSG) $\lambda: \mathbb{C}^{*} \rightarrow G$ such that $\lim _{t \rightarrow 0} \lambda(t) v=0$ ([Kraft 1984, Kap. III.2]). For a given 1-PSG $\lambda: \mathbb{C}^{*} \rightarrow G$ we define

$$
V(\lambda):=\left\{v \in V \mid \lim _{t \rightarrow 0} \lambda(t) v=0\right\} .
$$

Let $T \subset G$ be a maximal torus and denote by $X(T)$ the character group of $T$. Then we have the following weight decomposition

$$
V=\bigoplus_{\gamma \in X(T)} V_{\gamma}, \quad V_{\gamma}:=\{v \in V \mid \rho(t) v=\gamma(t) \cdot v \text { for } t \in T\} .
$$

$V_{\gamma}$ is called the weight space of weight $\gamma$. If $\lambda: \mathbb{C}^{*} \rightarrow T \subset G$ a 1-PSG of $T$, then

$$
V(\lambda)=\bigoplus_{\langle\lambda, \gamma\rangle>0} V_{\gamma}
$$

where $\langle\lambda, \gamma\rangle$ denotes the usual pairing between $X(T)$ and the group $Y(T)$ of 1PSG's of $T:\langle\lambda, \gamma\rangle=n$ if $\gamma(\lambda(t))=t^{n}$ for $t \in \mathbb{C}^{*}$.

Varying $\lambda \in Y(T)$ we find finitely many different subspaces $V(\lambda)$ of $V$. Using the Hilbert-Mumford criterion mentioned above and the fact that every 1-PSG of $G$ is conjugate to a 1-PSG of $T$, we obtain the following description of the nullcone of $V^{\oplus k}$ for any $k \geq 1$ :

$$
N_{V^{\oplus k}}=\bigcup_{\lambda \in Y(T)} G V(\lambda)^{\oplus k} .
$$

Moreover, $V(\lambda)$ is normalized by a parabolic subgroup $P(\lambda)$ containing $T$ which depends on $\lambda$ and on $V$. In fact,

$$
P(\lambda) \supseteq\left\{g \in G \mid \lim _{t \rightarrow 0} \lambda(t) g \lambda(t)^{-1} \text { exists }\right\},
$$


and the right-hand side is a parabolic subgroup with Levi subgroup $G^{\lambda}$ [Kempf 1978 , p. 305]. Clearly, we have equality for a suitably chosen $\lambda$. It follows that $G V(\lambda)$ is closed and that there is a proper surjective morphism

$$
G \times_{P(\lambda)} V(\lambda)^{\oplus k} \rightarrow G V(\lambda)^{\oplus k}
$$

where $G \times{ }_{P(\lambda)} V(\lambda)^{\oplus k}$ denotes the associated vector bundle to the principal bundle $G \rightarrow G / P$, i.e., $G \times{ }_{P(\lambda)} V(\lambda)^{\oplus k}:=\left(G \times V(\lambda)^{\oplus k}\right) / P(\lambda)$ with the diagonal action of $P(\lambda)$ given by $p(g, v):=\left(g p^{-1}, p v\right)$.

Let us denote by $X_{V} \subset X(T)$ the set of weights of $V$. Given any subset $X \subset X_{V}$ we put $V_{X}:=\oplus_{\gamma \in X} V_{\gamma}$.

Definition 1.1. A subset $X \subset X_{V}$ is called unstable if there is a $\lambda \in Y(T)$ such that $\langle\lambda, \alpha\rangle>0$ for all $\alpha \in X$, and maximal unstable if $X$ is maximal under this condition. We denote by $\Xi_{V}$ the set of maximal unstable subsets of $X_{V}$.

Clearly, we have

$$
N_{V^{\oplus k}}=\bigcup_{X \in \Xi_{V}} G V_{X}^{\oplus k}
$$

The Weyl group $W=N_{G}(T) / T$ acts on $X(T)$ and leaves $X_{V}$ invariant. If $X \subset X_{V}$ is maximal unstable then so is $w X$, and $G V_{X}=G V_{w X}$. Thus it suffices in (*) to choose a representative for any $W$-orbit of maximal unstable subsets of $X_{V}$. This representative can be chosen in such a way that $V_{X}$ is stable under a fixed Borel subgroup $B$ of $G$.

Definition 1.2. A subspace $U \subset V$ is called unstable if $U$ is annihilated by a 1PSG $\lambda$ (meaning that $U \subset V(\lambda)$ ), and maximal unstable if it is maximal under this condition. We denote by $U_{V}$ the set of maximal unstable subspaces of $V$.

If $X \subset X_{V}$ is a maximal unstable subset then $V_{X}$ is a maximal unstable subspace. Conversely, if $U \subset V$ is a maximal unstable subspace, then $U=g V_{X}$ for a suitable $g \in G$ and a maximal unstable subset $X \subset X_{V}$. Moreover, if $U$ is $T$-stable, then $U=V_{X}$ for some $X \in \Xi_{V}$.

We will consider $U_{V}$ as a $G$-stable subset of the Grassmannian:

$$
u_{V} \subset \operatorname{Gr}(V):=\bigcup_{1 \leq m \leq \operatorname{dim} V} \operatorname{Gr}_{m}(V) .
$$

In fact, $U_{V}$ consists of finitely many closed orbits since each maximal unstable subset $U$ is normalized by a parabolic subgroup.

In order to determine which spaces $V_{X}^{\oplus k}$ contribute to irreducible components of $N_{V^{\oplus k}}$ in the decomposition (*) we have to check whether or not $V_{X^{\prime}}^{\oplus k} \subset G V_{X}^{\oplus k}$. The next lemma is a first step in this direction. 
Lemma 1.3. Let $U \subset V$ be a $B$-stable subspace where $B \subset G$ is a Borel subgroup containing $T$. Assume that there is a $g \in G$ and a subset $X^{\prime} \subset X(T)$ such that $g U \subset \bigoplus_{\gamma \in X^{\prime}} V_{\gamma}$. Then $w X_{U} \subset X^{\prime}$ for a suitable $w \in W$.

Proof. The Bruhat Lemma implies that we can write $g$ in the form $g=u n_{w} b$ where $b \in B, n_{w} \in N_{G}(T)$ is a representative of some $w \in W$, and $u \in B_{u}$, the unipotent radical of $B$. Then $b U=U$ and so $U_{1}:=n_{w} b U$ is $T$-stable with weights $X_{U_{1}}=w X_{U}$. It follows that the subspace $g U=u U_{1}$ has the property that its projection onto every weight space $V_{\gamma}, \gamma \in w X_{U}$, is nonzero. On the other hand, we have $u U_{1}=g U \subset \bigoplus_{\gamma \in X^{\prime}} V_{\gamma}$, by assumption. This implies that $w X_{U} \subset X^{\prime}$.

For any representation $V$ of $G$ we define

$$
m(V):=\max \{\operatorname{dim} U \mid U \subset V \text { maximal unstable subspace }\} .
$$

We always have $m(V)<\operatorname{dim} V$. If $V$ is self-dual, meaning that $X_{V}=-X_{V}$, then

$$
m(V)=\frac{\operatorname{dim} V-\operatorname{dim} V_{0}}{2} .
$$

In fact, if $X$ is a maximal unstable subset of $X_{V}$ and $\gamma$ is a nonzero weight of $V$, then exactly one of $\gamma,-\gamma$ is contained in $X$.

Theorem 1.4. Let $V$ be a representation of $G$ and let $\left\{X_{1}, X_{2}, \ldots, X_{s}\right\}$ be a set of representatives of the $W$-orbits of maximal unstable subsets of $X_{V}$. If every irreducible subrepresentation $U$ of $V$ occurs at least $m(U)$ times, the closed subsets $C_{i}:=G V_{X_{i}}$ are the distinct irreducible components of the nullcone $N_{V}$. If $P_{i}$ denotes the normalizer of $V_{X_{i}}$, the canonical morphism $G \times_{P_{i}} V_{X_{i}} \rightarrow C_{i}$ is a resolution of singularities, i.e., it is proper and birational.

Proof. Assume that $V_{X_{i}} \subset G V_{X_{j}}$ for some $i \neq j$. Fix a decomposition of $V$ into irreducible factors:

$$
\phi: V \stackrel{\sim}{\rightarrow} \rightarrow \bigoplus_{\gamma} W_{\gamma}^{\oplus m_{\gamma}}
$$

where the $W_{\gamma}$ are pairwise nonisomorphic simple $G$-modules. Then $\phi$ induces isomorphisms $\phi_{i}: V_{X_{i}} \stackrel{\sim}{\rightarrow} \rightarrow \bigoplus_{\gamma} m_{\gamma} W_{\gamma_{X_{i}}}$ for all $i$. Since $m_{\gamma} \geq \operatorname{dim} W_{\gamma_{X_{i}}}$ we can find a vector $v \in V_{X_{i}}$ such that the components of $\phi(v)$ in the $m_{\gamma}$ copies of $W_{\gamma}$ span the subspace $W_{\gamma_{X_{i}}} \subset W_{\gamma}$ for every $\gamma$. By assumption, there is a $g \in G$ such that $g v \in V_{X_{j}}$. It follows that $g W_{\gamma_{X_{i}}} \subset W_{\gamma_{X_{j}}}$ for all $\gamma$, and so $g V_{X_{i}} \subset V_{X_{j}}$. Now we can apply Lemma 1.3 above to find a $w \in W$ such that $w X_{i} \subset X_{j}$. This contradiction proves the first part of the theorem.

For the second part we have already remarked that $P_{i} \subset G$ is a parabolic subgroup, that $C_{i} \subset N_{V}$ is closed and that the canonical morphism

$$
\eta_{i}: G \times{ }_{P_{i}} V_{X_{i}} \rightarrow C_{i}, \quad[g, v] \mapsto g v
$$


is proper and surjective. Now we use the same decomposition $\phi: V \stackrel{\sim}{\rightarrow} \rightarrow \bigoplus_{\gamma} W_{\gamma}^{m_{\gamma}}$ as above. It is easy to see that the vectors $v \in V_{X_{i}}$ with the property that the components of $\phi(v)$ in the $m_{\gamma}$ copies of $W_{\gamma}$ span the subspace $W_{\gamma_{X_{i}}}$ for all $\gamma$ form a dense open subset $Z_{i} \subset V_{X_{i}}$. For any $v \in Z_{i}$ we therefore have

$$
\operatorname{Stab}_{G} v=\operatorname{Stab}_{G} V_{X_{i}} \subset \operatorname{Norm}_{G} V_{X_{i}}=P_{i} .
$$

Thus, $\eta_{i}$ is injective on the dense subset $G \times{ }_{P_{i}} Z_{i}$ of $G \times{ }_{P_{i}} V_{X_{i}}$, hence birational.

Remark 1.5. Let $\Delta \subset X(T)$ be the root system, $B \supset T$ a Borel subgroup of $G$ and $\Delta^{+} \subset \Delta$ the corresponding positive roots. In order to determine the $W$ representatives $\left\{X_{1}, X_{2}, \ldots, X_{s}\right\}$ of the maximal unstable subsets it suffices to consider those subsets $X \subset X(T)$ where the corresponding subspace $V_{X}$ is invariant under $B$. This means that $X$ satisfies the condition

$$
\left(X+\Delta^{+}\right) \cap X_{V} \subset X .
$$

We will call these subsets $\Delta^{+}$-invariant or $B$-invariant.

Remark 1.6. If $p: V \rightarrow V^{\prime}$ is a surjective $G$-homomorphism then the induced morphism $p: N_{V} \rightarrow N_{V^{\prime}}$ is surjective. In particular, $N_{V^{\prime}}=\bigcup_{i} p\left(C_{i}\right)$ and the images $p\left(C_{i}\right)$ are all closed, but there might be some inclusions among the different $p\left(C_{i}\right)$.

The next result is an immediate consequence of the proof of Theorem 1.4. There is an obvious generalization to arbitrary representations $V$ whose formulation is left to the reader.

Theorem 1.7. Let $V$ be an irreducible representation of $G$ and let $\left\{X_{1}, \ldots, X_{s}\right\}$ be representatives of the $W$-orbits of maximal unstable subsets of $X_{V}$. For a given $k \geq$ 1 the corresponding component $G V_{X_{i}}^{\oplus k} \subset N_{V^{\oplus k}}$ is not visible (that is, it is contained in another component $G V_{X_{j}}^{\oplus k}$ ) if and only if the following condition holds:

$\left(\mathrm{C}_{k}\right) \quad$ For every $k$-dimensional subspace $U \subset V_{X_{i}}$ there exist an element $g \in G$ and an index $j \neq i$ such that $g U \subset V_{X_{j}}$.

\section{Examples}

In this section we give a number of important examples that motivated the study of the nullcone of multiple copies of a given representation.

Example 2.1 (Adjoint representations). Let $\mathfrak{g}:=$ Lie $G$ be the Lie algebra of $G$. Then $N_{\mathfrak{g}}$ is the set of nilpotent elements in $\mathfrak{g}$. Fixing a Borel subgroup $B$ of $G$ we see that there is a unique maximal $B$-invariant unstable subspace in $\mathfrak{g}$, namely the nilradical $\mathfrak{n}$ of Lie $B$. The corresponding $X_{\mathfrak{n}}$ is the set of positive roots. This implies: 
For any $k \geq 1$ the nullcone $N_{\mathfrak{g}^{\oplus k}}$ is irreducible and

$$
G \times{ }_{B} \mathfrak{n}^{\oplus k} \rightarrow N_{\mathfrak{g}^{\oplus k}}
$$

is a resolution of singularities. Moreover, $\operatorname{dim} N_{\mathfrak{g}^{\oplus k}}=\frac{1}{2}(k+1)(\operatorname{dim} G-\mathrm{rk} G)$.

Example 2.2 (Orthogonal representations). Let $G:=\mathrm{SO}_{n}$ be the special orthogonal group and $V:=\mathbb{C}^{n}$ the standard representation. We claim that for odd $n$ there is a unique maximal $B$-invariant unstable subset whereas for even $n$ there are two. More precisely:

Let $V$ be the standard representation of the orthogonal group $\mathrm{SO}_{n}$.

(1) If $n=2 m+1 \geq 3$ is odd then $N_{V^{\oplus k}}$ is irreducible for any number $k$ of copies of $V$.

(2) If $n=2 m \geq 2$ is even then $N_{V^{\oplus k}}$ is irreducible for $k<m$ and has two irreducible components for $k \geq m$, permuted by $O_{n}$.

In both cases $\operatorname{dim} N_{V}=n-1$ and $\operatorname{dim} N_{V^{\oplus k}} \leq\left(\begin{array}{c}m \\ 2\end{array}\right)+k m$ with equality for $k \geq m$.

Proof. (1) For $n=2 m+1$ the weights of $V$ are $\left\{ \pm \varepsilon_{1}, \pm \varepsilon_{2}, \ldots, \pm \varepsilon_{m}, 0\right\}$. Since the positive roots $\Delta^{+}$contain the elements $\left\{\varepsilon_{i}-\varepsilon_{j} \mid i<j\right\}$ and $\left\{\varepsilon_{k} \mid 1 \leq k \leq m\right\}$ it easily follows that there is exactly one maximal $\Delta^{+}$-invariant unstable subset, namely $\left\{\varepsilon_{1}, \varepsilon_{2}, \ldots, \varepsilon_{m}\right\}$.

(2) For $n=2 m$ the weights are $\left\{ \pm \varepsilon_{1}, \pm \varepsilon_{2}, \ldots, \pm \varepsilon_{m}\right\}$ and the positive roots $\Delta^{+}$ contain the elements $\left\{\varepsilon_{i} \pm \varepsilon_{j} \mid i<j\right\}$. It follows that there are two maximal $\Delta^{+}$invariant unstable subsets, $\left\{\varepsilon_{1}, \varepsilon_{2}, \ldots, \varepsilon_{m}\right\}$ and $\left\{\varepsilon_{1}, \varepsilon_{2}, \ldots, \varepsilon_{m-1},-\varepsilon_{m}\right\}$, which are not equivalent under the Weyl group. The corresponding subspaces $U$ and $U^{\prime}$ are both totally isotropic and are equivalent under $O_{n}$, but not under $\mathrm{SO}_{n}$. On the other hand, $\mathrm{GL}(U)$ can be identified with a Levi subgroup of $\mathrm{SO}_{n}$ and so every linear automorphism of $U$ (or $U^{\prime}$ ) is induced by an element of $\mathrm{SO}_{n}$. Hence, for every linear subspaces $W$ of $U^{\prime}$ of dimension $<m$ there is an element $g \in \mathrm{SO}_{n}$ such that $g W \subset U \cap U^{\prime} \subset U$. This proves the claim by Theorem 1.7.

The statement about dimensions follows from the fact that the normalizer of $U$ is the maximal parabolic subgroup $P$ with Levi factor $\mathrm{GL}_{m}$ whose codimension in $\mathrm{SO}_{n}$ is $\left(\begin{array}{c}m \\ 2\end{array}\right)$.

Example 2.3 (Quadratic forms). (This example was worked out by Matthias Bürgin [2005].) Let $G=\mathrm{SL}_{3}$ with positive roots $\Delta^{+}=\left\{\varepsilon_{1}-\varepsilon_{2}, \varepsilon_{1}-\varepsilon_{3}, \varepsilon_{2}-\varepsilon_{3}\right\}$, acting linearly on $V:=S^{2}\left(\mathbb{C}^{3}\right)^{*}$, the quadratic forms in 3 variables.

There are two maximal $\Delta^{+}$-invariant unstable subsets, and the corresponding two components of the nullcone are already seen in 2 copies $V \oplus V$. The nullcone $N_{V}$ is an irreducible hypersurface of dimension 5 , and, for $k \geq 2, N_{V^{\oplus k}}$ is equidimensional with two components of dimension $2+3 k$. 
Proof. The weights of $V$ are $\left\{\varepsilon_{i}+\varepsilon_{j} \mid 1 \leq i \leq j \leq 3\right\}$ and one easily finds the following two maximal $\Delta^{+}$-invariant unstable subsets: $X_{1}:=\left\{2 \varepsilon_{1}, 2 \varepsilon_{2}, \varepsilon_{1}+\varepsilon_{2}\right\}$ and $X_{2}:=\left\{2 \varepsilon_{1}, \varepsilon_{1}+\varepsilon_{2}, \varepsilon_{1}+\varepsilon_{3}\right\}$. Note that $X_{2}$ is $W$-equivalent to the complement $X_{V} \backslash X_{1}$. The corresponding subspaces of $V$ are $V_{1}:=\left\langle x_{1}^{2}, x_{1} x_{2}, x_{2}^{2}\right\rangle$ and $V_{2}:=x_{1} \cdot\left\langle x_{1}, x_{2}, x_{3}\right\rangle$. It follows that the 2-dimensional subspace $\left\langle x_{1}^{2}, x_{2}^{2}\right\rangle \subset V_{1}$ is not equivalent to a subspace of $V_{2}$, because it contains two linearly independent squares. Similarly, one sees that the subspace $\left\langle x_{1} x_{2}, x_{1} x_{3}\right\rangle \subset V_{2}$ is not equivalent to a subspace of $V_{1}$. Thus both components are already seen in two copies (Theorem 1.7). The normalizers of $V_{1}$ and $V_{2}$ are the two parabolic subgroups $P_{1}$ and $P_{2}$ of codimension 2. Since the nullcone of one copy has codimension 1, hence dimension $5=2+3$ this implies that for all $k \geq 1$ the morphisms $G \times{ }^{P_{i}} V_{i}^{\oplus k} \rightarrow G V_{i}^{\oplus k}$ are of finite degree and so $\operatorname{dim} N_{V^{\oplus k}}=2+3 k$.

Example 2.4 (The case of 3-qubits). Take

$$
G:=\mathrm{SL}_{2} \times \mathrm{SL}_{2} \times \mathrm{SL}_{2} \quad \text { and } \quad V:=\mathbb{C}^{2} \otimes \mathbb{C}^{2} \otimes \mathbb{C}^{2},
$$

with the usual linear action of $G$. The positive roots are given by $\Delta^{+}=\{\alpha=$ $\left.2 \varepsilon_{1}, \alpha^{\prime}=2 \varepsilon_{1}^{\prime}, \alpha^{\prime \prime}=2 \varepsilon^{\prime \prime}\right\}$.

There are four maximal $\Delta^{+}$-invariant unstable subsets, three of them are permuted by the obvious action of $S_{3}$ on $V$ and hence on $X_{V}$. The nullcones $N_{V}$ and $N_{V^{\oplus 2}}$ are both irreducible, of dimension 7 and 11, respectively. The nullcone of $k \geq 3$ copies has 4 components, one of dimension $3+4 k$ which is stable under $S_{3}$ and three of dimension $1+4 k$ which are permuted by $S_{3}$.

Proof. Setting $\mathbb{C}^{2}=\mathbb{C} e_{0} \oplus \mathbb{C} e_{1}$ we get the basis $\left(e_{i j k}:=e_{i} \otimes e_{j} \otimes e_{k} \mid i, j, k \in\{0,1\}\right)$ of $V$. Since $\varepsilon_{1}=-\varepsilon_{0}$ for the weights on $\mathbb{C}^{2}$ the corresponding weights $\varepsilon_{i j k}$ of $V$ are given by the vertices of a cube. It is easy to see that the maximal $\Delta^{+}$-invariant unstable subsets of $X_{V}=\left\{\varepsilon_{i j k}\right\}$ are

$$
\begin{aligned}
& X_{0}:=\left\{\varepsilon_{000}, \varepsilon_{001}, \varepsilon_{010}, \varepsilon_{100}\right\}, \\
& X_{1}:=\left\{\varepsilon_{000}, \varepsilon_{001}, \varepsilon_{010}, \varepsilon_{011}\right\}, \\
& X_{2}:=\left\{\varepsilon_{000}, \varepsilon_{001}, \varepsilon_{100}, \varepsilon_{101}\right\}, \\
& X_{3}:=\left\{\varepsilon_{000}, \varepsilon_{010}, \varepsilon_{100}, \varepsilon_{110}\right\} .
\end{aligned}
$$

$X_{0}$ consists of the vertices of all edges containing $\varepsilon_{000}$ and $X_{1}, X_{2}, X_{3}$ correspond to the 3 faces with vertex $\varepsilon_{000}$. The latter are permuted by the action of $S_{3}$ on the weights $\varepsilon_{i j k}$ given by permuting the indices, and $X_{0}$ is invariant under $S_{3}$.

The normalizer of $V_{X_{0}}$ is the Borel subgroup $B \times B \times B$, and $V_{X_{1}}=e_{0} \otimes \mathbb{C}^{2} \otimes \mathbb{C}^{2}$ is normalized by $B \times \mathrm{SL}_{2} \times \mathrm{SL}_{2}$; similarly for $V_{X_{2}}$ and $V_{X_{3}}$. The claim now follows from the next lemma together with Theorem 1.7. The statements about dimensions follow in a similar way as in Example 2.3. 
Lemma 2.5. (1) Every 2-dimensional subspace $U$ of $V_{X_{1}}$ can be mapped into $V_{X_{1}} \cap V_{X_{0}}$ with an element of $B \times \mathrm{SL}_{2} \times \mathrm{SL}_{2}$.

(2) A generic 3-dimensional subspace of $V_{X_{1}}$ cannot be mapped into $V_{X_{0}}$ with an element of $\mathrm{SL}_{2} \times \mathrm{SL}_{2} \times \mathrm{SL}_{2}$.

Proof. (1) Recall that the representation of $\mathrm{SL}_{2} \times \mathrm{SL}_{2}$ on $V_{X_{1}} \simeq \mathbb{C}^{2} \otimes \mathbb{C}^{2}$ corresponds to the standard representation of $\mathrm{SO}_{4}$ on $\mathbb{C}^{4}$ where the invariant form on $\mathbb{C}^{2} \otimes \mathbb{C}^{2}$ is given by

$$
\left(e_{i j}, e_{i^{\prime} j^{\prime}}\right)= \begin{cases}1 & \text { if } i+i^{\prime}=j+j^{\prime}=1, \\ 0 & \text { otherwise. }\end{cases}
$$

Thus every 2-dimensional subspace of $V_{X_{1}}$ is perpendicular to an isotropic vector. Since $\mathrm{SO}_{4}$ acts transitively on the isotropic vectors, this implies that every 2dimensional subspace of $V_{X_{1}}$ can be mapped into the subspace perpendicular to the highest weight vector corresponding to $\varepsilon_{000}$ which is $V_{X_{1}} \cap V_{X_{0}}=e_{0} \otimes\left\langle e_{00}, e_{01}, e_{10}\right\rangle$.

(2) Let $U \subset V_{X_{1}}$ be any 3-dimensional subspace which is orthogonal to a nonisotropic vector. Then $h U \neq V_{X_{1}} \cap V_{X_{0}}$ for any $h \in \mathrm{SO}_{4}$ and so $h U$ always contains a vector whose coordinate in the weight space of $\mathbb{C} e_{011}$ is nonzero. But this implies that for any $g \in G$ the image $g U$ contains a vector whose projection into the subspace $V_{\left\{\varepsilon_{011}, \varepsilon_{111}\right\}} \subset V$ is nonzero. Thus $g U \nsubseteq V_{X_{0}}$ for all $g \in G$.

\section{Nullcones for $\theta$-representations}

Let $V$ be a representation of a reductive group $G$, and let $K \subset G$ be a reductive subgroup and $W \subset V$ a $K$-stable subspace. We have an induced morphism $W / / K \rightarrow V / / G$. This morphism is finite if and only if we have the following relation between the nullcone $N_{W}$ of $W$ with respect to $K$ and the nullcone $N_{V}$ of $V$ with respect to $G$ :

$$
N_{W}=N_{V} \cap W .
$$

Equivalently, the restrictions of the $G$-invariants on $V$ define a subalgebra $A$ of $\mathcal{O}(W)^{K}$ such that $\mathcal{O}(W)^{K}$ is a finitely generated $A$-module. There are well-known examples where this holds.

Examples 3.1. (1) Let $G$ be semisimple, $\mathfrak{g}=$ Lie $G$ the adjoint representation, $K:=\operatorname{Norm}_{G} T$ the normalizer of a maximal torus $T$ in $G$ and $\mathfrak{h}:=\operatorname{Lie} T$; then, by Chevalley's restriction theorem, $\mathfrak{h} / / K \stackrel{\sim}{\rightarrow} \rightarrow \mathfrak{g} / / G$, so $\mathcal{O}(\mathfrak{g})^{G}=\mathcal{O}(\mathfrak{h})^{K}$.

(2) Let $G v=\overline{G v} \subset V$ be a closed orbit and denote by $G_{v}$ the stabilizer of $v$. Define $K:=\operatorname{Norm}_{G} G_{v}$ and set $W:=V^{G_{v}}$. Then the induced morphism $W / / K \rightarrow V / / G$ is a closed immersion. In particular, $\left.\mathcal{O}(V)^{G}\right|_{W}=\mathcal{O}(W)^{K}$, that is, $\mathcal{O}(W)^{K}$ is generated by the restrictions of the $G$-invariants of $V$ to $W$. This generalization of (1) is due to Luna and Richardson [1979]. 
(3) Let $G$ be a semisimple group and $\theta$ an automorphism of finite order of $G$. Define $G^{\theta}:=\{g \in G \mid \theta(g)=g\}$. The automorphism $\theta$ defines an automorphism of $\mathfrak{g}:=$ Lie $G$, also denoted by $\theta$. Let $W \subset \mathfrak{g}$ be an eigenspace of $\theta$ and consider $W$ as a representation of $G^{\theta}$. Then $N_{\mathfrak{g}} \cap W=N_{W}$, see [Vinberg $1975 ; 1976]$.

Question 3.2. If such a "restriction property" holds for the pair $(G, V) \supset(K, W)$, does it also hold for two or more copies of $V$ and $W$ ? That is, do we have

$$
N_{V^{\oplus m}} \cap W^{\oplus m}=N_{W^{\oplus m}} \quad \text { for } m \geq 2 ?
$$

Among the examples above, this holds for (1). It is even true that $\left.\mathscr{O}\left(\mathfrak{g}^{n}\right)^{G}\right|_{\mathfrak{h}^{n}}$ coincides with $\mathcal{O}\left(\mathfrak{h}^{n}\right)^{K}$, but this is a difficult theorem of Joseph [1997] (compare [Wallach 1993]). This stronger result does not carry over to the generalization (2), but it might still be true that $(* *)$ holds. Concerning the last example we will now show that $(* *)$ holds in a more general situation.

Theorem 3.3. Let $\rho: G \rightarrow \mathrm{GL}(V)$ be a representation of a reductive group $G$ and let $\theta$ be a semisimple automorphism of $V$ which normalizes $\rho(G)$. Consider the subgroup $K:=\{h \in G \mid \rho(h) \theta=\theta \rho(h)\}$, and let $W \subset V$ be an eigenspace of $\theta$. Then $K$ is reductive and $N_{V} \cap W=N_{W}$. In particular, the induced morphism $W / / K \rightarrow V / / G$ is finite.

Proof. It is well-known that $K$ is reductive. A short argument can be found in [Kraft et al. 1999, Corollary 2] where we show that a closed subgroup $K \subset G$ of a reductive group $G$ is reductive if and only if $\operatorname{Lie} H$ has an $\operatorname{Ad}(H)$-stable complement in Lie $G$.

Since $N_{W} \subset N_{V}$, it remains to show that $N_{V} \cap W \subseteq N_{W}$. Multiplying $\theta$ with a suitable scalar we can assume that $W=V^{\theta}$. Define $S:=\overline{\langle\theta\rangle} \subset \operatorname{GL}(V)$, the closure of the subgroup generated by $\theta$. This group $S$ is commutative and reductive, normalizes $\rho(G)$ and acts trivially on $W$. Therefore, $\tilde{G}:=S \cdot \rho(G) \subset \operatorname{GL}(V)$ is again reductive. Since $\tilde{K}:=\operatorname{Cent}_{\tilde{G}}(S)$ has the same image in $\operatorname{GL}(W)$ as $K$ and since the nullcone of $V$ with respect to $\tilde{G}$ contains $N_{V}$, it suffices to prove the claim with $G$ replaced by $\tilde{G}$ and $K$ replaced by $\tilde{K}$. But now we are in the situation of [Kempf 1978, Corollary 4.5], which implies that for every point in $x \in N_{V} \cap W$ there is a 1-PSG $\lambda$ of $\tilde{K}$ such that $\lim _{t \rightarrow 0} \lambda(t) x=0$.

From now on we will consider the following special case which was studied in detail in [Vinberg 1976]. Let $G$ be a connected reductive group, $\mathfrak{g}:=$ Lie $G$ its Lie algebra, $\theta$ a semisimple automorphism of $G$ and $K:=G^{\theta}$ the fixed point group. We know that $K$ is reductive, but not necessarily connected. The automorphism $\theta$ induces an automorphism of the Lie algebra $\mathfrak{g}$, also denoted by $\theta$, and $\mathfrak{g}^{\theta}=\mathfrak{k}:=$ Lie $K$. Moreover, every eigenspace $V \subset \mathfrak{g}$ of $\theta$ is a representation of $K$. We fix a 
$\theta$-stable Cartan subalgebra $\mathfrak{t} \subset \mathfrak{g}$ such that $\mathfrak{t}_{0}:=\mathfrak{t} \cap \mathfrak{k}$ is a Cartan subalgebra of $\mathfrak{k}$ and denote the corresponding maximal tori by $T \subset G$ and $T_{0} \subset K$.

If $\mathfrak{b} \subset \mathfrak{g}$ is a $\theta$-stable Borel subalgebra, then the intersection of the nilradical $\mathfrak{n}_{\mathfrak{b}}$ of $\mathfrak{b}$ with an eigenspace $V$ of $\theta$ is an unstable subspace of $V$. We will see that all maximal unstable subspaces are obtained in this way.

Fix a Borel subalgebra $\mathfrak{b}_{0} \subset \mathfrak{k}$ containing $\mathfrak{t}_{0}$ and define the following set of unstable subspaces:

$\mathscr{B}_{V}:=\left\{V_{\mathfrak{b}}:=\mathfrak{n}_{\mathfrak{b}} \cap V \mid \mathfrak{b}\right.$ a $\theta$-stable Borel subalgebra of $\mathfrak{g}$ containing $\mathfrak{b}_{0}$ and $\left.\mathfrak{t}\right\}$

Proposition 3.4. Let $V \subset \mathfrak{g}$ be an eigenspace of $\theta$. Then the maximal elements of $\mathscr{B}_{V}, \mathscr{B}_{V}^{\max }$, form a set of representatives of the $K^{0}$-equivalence classes of maximal unstable subspaces of $V$. In particular, for any number $n$ of copies of $V$ we have

$$
N_{V^{\oplus n}}=\bigcup_{U \in \mathscr{B}_{V}^{\max }} K^{0} U^{\oplus n}
$$

Proof. If $U \subset V$ is an unstable subspace annihilated by the 1-PSG $\lambda$ of $T_{0}$ we can assume that $\lambda$ is regular as a 1-PSG of $T$. This follows easily from the fact that $\mathfrak{t}^{\text {reg }} \cap \mathfrak{t}_{0}$ is open and dense in $\mathfrak{t}_{0}$ (see [Borel and Mostow 1955]). Then $\mathfrak{n}:=\{x \in$ $\left.\mathfrak{g} \mid \lim _{t \rightarrow 0} \lambda(t) x=0\right\}$ is the nilpotent radical of a $\theta$-stable Borel subalgebra $\mathfrak{b}$, and $\mathfrak{n} \cap V$ is unstable and contains $U$, by construction. By conjugation with a suitable element of $K^{0}$ we can assume that $\mathfrak{b}$ contains $\mathfrak{b}_{0}$. Thus every maximal unstable subspace is conjugate to a maximal element from $\mathscr{B}_{V}$.

Let $B_{0} \subset K$ be the Borel subgroup with Lie algebra $\mathfrak{b}_{0}$. If two maximal subspaces $U_{1}, U_{2} \in \mathscr{B}_{V}$ are equivalent under $K^{0}$ then there is an element $n \in \operatorname{Norm}_{K^{0}}\left(T_{0}\right)$ which sends $U_{1}$ onto $U_{2}$. This implies that $U_{2}$ is stable under $B_{0}$ and $n B_{0} n^{-1}$. Hence the normalizer of $U_{2}$ contains the subgroup $H$ generated by $B_{0}$ and $n B_{0} n^{-1}$. Since $H$ contains $n$ we get $U_{1}=U_{2}$.

Question 3.5. Which maximal unstable subspaces from $\mathscr{B}_{V}$ contribute to irreducible components of $N_{V}$, and how many components do we have?

One knows that $N_{V}$ is a complete intersection and contains only finitely many $K$-orbits. More precisely, the invariant ring of $V$ is generated by algebraically independent elements, the quotient morphism $\pi: V \rightarrow V / / K$ is flat and each fiber of $\pi$ contains only finitely many orbits (see [Vinberg 1976]).

Since $\theta$ normalizes $\mathfrak{t}$ we have an action of $\theta$ on the Weyl group $W:=W(G, T)$. Denote by $W^{\theta} \subset W$ the subgroup of elements fixed by $\theta$. It is easy to see that $W^{\theta}$ acts simply transitively on the set of $\theta$-stable Borel subalgebra containing $\mathfrak{t}$. Therefore, the number of $\theta$-stable Borel subalgebra containing $\mathfrak{t}$ and $\mathfrak{b}_{0}$ is given by the index $\left[W^{\theta}: W_{K}\right]$ where $W_{K}:=W\left(K^{0}, T_{0}\right)$. (Recall that the restriction of $W^{\theta}$ to $\mathfrak{t}_{0}$ is injective and the image contains $W_{K}$.) 
Proposition 3.6. We use the notation introduced above.

(1) We have

$$
\# \mathscr{B}_{V}^{\max } \leq \# \mathscr{B}_{V} \leq\left[W^{\theta}: W_{K}\right] .
$$

(2) Assume that $\theta$ has order 2 and let $V \subset \mathfrak{g}$ be the (-1)-eigenspace, so that $\mathfrak{g}=\mathfrak{k} \oplus V$. Then

$$
\# \mathscr{B}_{V}^{\max }=\# \mathscr{B}_{V}=\left[W^{\theta}: W_{K}\right] .
$$

Proof. (1) follows from what was said above. For (2) we remark that under the given assumptions we have $\mathfrak{b}=\left(\mathfrak{t}+\mathfrak{b}_{0}\right) \oplus V_{\mathfrak{b}}$ and so $\mathfrak{b}$ is determined by $V_{\mathfrak{b}}$, and all $V_{\mathfrak{b}}$ have the same dimension, hence are maximal.

Example 3.7. [Quiver representations of type $\tilde{A}_{1}$ ] Let $U, W$ be two finite dimensional vector spaces and set $G:=\operatorname{GL}(U \oplus W)$ and $\theta:=\operatorname{Int}\left(\operatorname{id}_{U},-\operatorname{id}_{W}\right)$, so $\theta$ is the conjugation with $\left[\begin{array}{ll}\mathrm{id}_{U} & \\ & -\mathrm{id}_{W}\end{array}\right]$. Then $K=\mathrm{GL}(U) \times \mathrm{GL}(W)$ and $V=$ $\operatorname{Hom}(U, W) \oplus \operatorname{Hom}(W, U)$ with the obvious linear action of $K$. By Proposition 3.6(2) we get $\# \mathscr{B}_{V}=\left(\begin{array}{c}\operatorname{dim} U+\operatorname{dim} W \\ \operatorname{dim} U\end{array}\right)$ and all unstable spaces in $\mathscr{B}_{V}$ are maximal and nonequivalent. It is known that $N_{V}$ is irreducible for $m \neq n$ and has 2 irreducible components for $m=n$. A detailed analysis of the geometry of this representation can be found in [Kempken 1982].

Remark 3.8. Let $\mathfrak{a} \subset V$ be a Cartan subspace, i.e., a maximal subspace consisting of semisimple elements. Then $K \mathfrak{a}$ is dense in $V$ and the inclusion induces an isomorphism $\mathfrak{a} / / W_{V} \stackrel{\sim}{\rightarrow} \rightarrow V / / K^{0}$ where $W_{V}:=\operatorname{Norm}_{K^{0}}(\mathfrak{a}) / \operatorname{Cent}_{K^{0}}(\mathfrak{a})$ is a finite group generated by reflections; see [Vinberg 1976]. If $\operatorname{dim} \mathfrak{a}=\operatorname{dim} \mathfrak{t}=\operatorname{rk} G$, then $\operatorname{Norm}_{K^{0}}(\mathfrak{a})$ is finite since $\mathfrak{n o r m}_{\mathfrak{k}}(\mathfrak{a})=\mathfrak{n o r m}_{\mathfrak{g}}(\mathfrak{a}) \cap \mathfrak{k}=\mathfrak{a} \cap \mathfrak{k}=\{0\}$, and so $\operatorname{dim} V=$ $\operatorname{dim} K+\operatorname{dim} \mathfrak{a}$. It follows that in this case the generic orbits in $V$ have finite stabilizer.

We add here a useful criterion to decide if an element $v=\left(v_{1}, v_{2}, \ldots, v_{k}\right) \in V^{\oplus k}$ belongs to the nullcone. A special case of this result played a fundamental rôle in the determination of the Hilbert series for the invariants of pairs of 4-qubits (see [Wallach 2005] and Section 4 below).

First of all, it is well-known and easy to see that a $k$-tuple $v=\left(v_{1}, v_{2}, \ldots, v_{k}\right)$ belongs to the nullcone of $V^{\oplus k}$ if and only if the subalgebra $\mathfrak{s} \subset \mathfrak{g}$ generated by $v_{1}, v_{2}, \ldots, v_{k}$ consists of nilpotent elements. (One simply uses the fact that $v=\left(v_{1}, v_{2}, \ldots, v_{k}\right)$ is annihilated by a 1-PSG $\lambda$ if and only if the subalgebra $\mathfrak{s}$ is annihilated by $\lambda$.)

Proposition 3.9. For a given $k \geq 1$ let $v=\left(v_{1}, v_{2}, \ldots, v_{k}\right) \in V^{\oplus k}$ and denote by $\mathfrak{s} \subset \mathfrak{g}$ the Lie algebra generated by $v_{1}, v_{2}, \ldots, v_{k}$. Then $v$ belongs to the nullcone $N_{V^{\oplus k}}$ if and only if the following conditions hold:

(1) $v_{1}, v_{2}, \ldots, v_{k} \in \mathfrak{g}$ are nilpotent elements.

(2) The Lie algebra $\mathfrak{s} \cap \mathfrak{k}$ is nilpotent. 
Proof. One direction of the claim follows from the observation above. Now assume that the elements $v_{i} \in \mathfrak{g}$ are nilpotent and that $\mathfrak{s} \cap \mathfrak{k}$ is a nilpotent Lie algebra. By construction, $\mathfrak{s}$ is $\theta$-invariant and $\mathfrak{s} \cap \mathfrak{k}=\mathfrak{s}^{\theta}$. Let $\mathfrak{r}$ be the radical of $\mathfrak{s}$. Then $\theta$ induces a semisimple automorphism of $\mathfrak{s} / \mathfrak{r}$. The main result of [Borel and Mostow 1955], implies that if $\mathfrak{s} / \mathfrak{r} \neq 0$ then the induced automorphism fixes a regular semisimple element. This implies that $\mathfrak{s}=\mathfrak{r}$ so it is solvable. But then it consists of nilpotent elements since it is generated by nilpotent elements.

From now on let us assume that $\theta$ has order 2 and let $V$ be the (-1)-eigenspace, so that $\mathfrak{g}=\mathfrak{k} \oplus V$. This is the situation studied in [Kostant and Rallis 1971].

Remark 3.10. If we choose $G$ to be the adjoint group (and $\theta$ of order 2) it is shown in [Kostant and Rallis 1971] that $K$ has a dense orbit in the nullcone $N_{V}$. As a consequence, the number of irreducible components of $N_{V}$ is less or equal to the order of the component group $K / K^{0}$. As an example let us look at the quiver representations of type $\tilde{A}_{1}$ from Example 3.7. If $U=W$ and if we replace $G:=\operatorname{GL}(U \oplus U)$ by its image $\bar{G}$ in $\operatorname{Aut}(\operatorname{End}(U \oplus U))$ then $\bar{K}:=\bar{G}^{\theta}$ contains the image of the element $\left[{ }_{\text {id }}\right.$ id $]$ which permutes the two copies of $\operatorname{End}(U)$ in the representation of $\bar{K}$ on $V:=\operatorname{End}(U) \oplus \operatorname{End}(U)$. In particular, $\bar{K}$ has a dense orbit in the (reducible) nullcone $N_{V}$, and $\bar{K} / \bar{K}^{0}$ has order 2 .

Proposition 3.11. Assume that $V$ contains a Cartan subalgebra of $\mathfrak{g}$. Let $V_{\mathfrak{b}} \in$ $\Re_{V}$ be a maximal unstable subspace. Then $K^{0} V_{\mathfrak{b}}$ is an irreducible component of $N_{V}$ if and only if $V_{\mathfrak{b}}$ contains a regular nilpotent element of $\mathfrak{g}$. Any two different subspaces of $\mathscr{T}_{V}$ of this form define different irreducible components.

Proof. We first show that $V$ contains a regular nilpotent element $n$. Let $\mathfrak{a} \subset V$ be a Cartan subalgebra of $\mathfrak{g}$ and choose a Borel subalgebra $\mathfrak{b} \supset \mathfrak{a}$. Denote by $\left(\alpha_{1}, \ldots, \alpha_{\ell}\right)$ the corresponding simple roots and fix nonzero elements $e_{i} \in \mathfrak{g}_{\alpha_{i}}$. Since $\left.\theta\right|_{\mathfrak{a}}=-\mathrm{id}_{\mathfrak{a}}$ we see that $\theta\left(\mathfrak{g}_{\alpha}\right)=\mathfrak{g}_{-\alpha}$ for any root $\alpha$. Choose $h \in \mathfrak{a}$ such that $\left[h, e_{i}\right]=2 e_{i}$ for all $i$. If $e:=\sum_{i} a_{i} e_{i}$ and $f:=\theta(e)=\sum_{i} a_{i} f_{i}$, where $f_{i}=\theta\left(e_{i}\right) \in \mathfrak{g}_{-\alpha_{i}}$, then we have $[h, f]=-2 f$. Moreover, we can solve the equation $[e, f]=h$ because $[e, f]=\sum_{i} a_{i}^{2}\left[e_{i}, f_{i}\right]$ and the elements $h_{i}:=\left[e_{i}, f_{i}\right]$ form a basis of $\mathfrak{a}$. It follows that $(e, f, h)$ is an $\theta$-stable $\mathfrak{s l}_{2}$-triple. In particular, the element $n:=h+e-f$ belongs to $V$ and is nilpotent, hence conjugate to $e$.

As a consequence, we see that the regular nilpotent elements in $N_{V}$ form a nonempty open set. Since $K$ has a dense orbit $O_{K}$ in $N_{V}$ (see Remark 3.10) it follows that $O_{K}$ consists of regular nilpotent elements. Hence every irreducible component contains a dense set of regular nilpotent elements. Thus $K^{0} V_{\mathfrak{b}}$ is an irreducible component of $N_{V}$ if and only if $V_{\mathfrak{b}}$ contains a regular nilpotent element.

It remains to see that different subsets $V_{\mathfrak{b}}$ containing regular nilpotent elements define different irreducible components of $N_{V}$. So assume that $K^{0} V_{\mathfrak{b}}=K^{0} V_{\mathfrak{b}^{\prime}}$ 
and let $x \in V_{\mathfrak{b}}$ be a regular nilpotent element. Then there is a $k \in K^{0}$ such that $x^{\prime}:=k x \in V_{\mathfrak{b}^{\prime}}$. It follows that the regular nilpotent element $x^{\prime}$ belongs to $\mathfrak{b}^{\prime}$ and also to $k \mathfrak{b} k^{-1}$. Thus $\mathfrak{b}^{\prime}=k \mathfrak{b} k^{-1}$ since a regular nilpotent element belongs to a unique Borel subalgebra. It follows that $k \mathfrak{b}_{0} k^{-1}=\mathfrak{b}_{0}$, hence $k \in B_{0}$ and so $\mathfrak{b}=\mathfrak{b}^{\prime}$.

In fact, all elements from $O_{K}$ have finite stabilizer as we have mentioned above. In order to see that $K_{x}$ is finite for a regular nilpotent $x \in V_{\mathfrak{b}} \subset V$ we first remark that $\left(G_{x}\right)^{0} \subset B_{x}$ and so $\left(K_{x}\right)^{0} \subset\left(B_{0}\right)_{x}$. Moreover, $B x$ is dense in $n_{\mathfrak{b}}$, hence $\mathfrak{n}_{\mathfrak{b}}=[\mathfrak{b}, x]=\left[\mathfrak{b}_{0}, x\right]+[V, x]$ which implies that $\left[\mathfrak{b}_{0}, x\right]=\mathfrak{n}_{\mathfrak{b}} \cap V=V_{\mathfrak{b}}$, in other words $B_{0} x$ is dense in $V_{\mathfrak{b}}$. But $\operatorname{dim} V_{\mathfrak{b}}=\operatorname{dim} \mathfrak{n}-\operatorname{dim} \mathfrak{n}_{0}=\operatorname{dim} K-\operatorname{dim} \mathfrak{n}_{0}=\operatorname{dim} B_{0}$ and so the stabilizer of $x$ in $B_{0}$ is finite.

Remark 3.12. It is easy to see that the regular nilpotent elements in $V$ form a single $K$-orbit.

In addition to the assumptions of Proposition 3.11, assume that $\mathrm{rk} G=\operatorname{rk} K$. Then $\mathfrak{t}=\mathfrak{t}_{0}$ and so $V$ is a sum of weight spaces. This implies a combinatorial description of the irreducible components of $N_{V}$ :

Corollary 3.13. Assume that $V$ contains a Cartan subalgebra of $\mathfrak{g}$ and that $G, K$ have equal rank. Then $K^{0} V_{\mathfrak{b}}$ is an irreducible component of $N_{V}$ if and only if $V_{\mathfrak{b}}$ contains all simple root spaces with respect to $\mathfrak{b}$.

Example 3.14 (Symmetric matrices). Consider the automorphism $\theta: A \mapsto\left(A^{t}\right)^{-1}$ of $G:=\mathrm{SL}_{n}$. Then $K:=G^{\theta}=O_{n}$ and $V:=\left(\mathfrak{s l}_{n}\right)_{-1}$ is the space of "traceless" symmetric $n \times n$-matrices which can be identified with $S^{2}\left(\mathbb{C}^{n}\right) / \mathbb{C}$. Clearly, $V$ contains a Cartan subalgebra of $\mathfrak{s l}_{n}$, given by the diagonal matrices. Hence, we can apply Corollary 3.13 and obtain:

Proposition 3.15. Consider the representation of $\mathrm{SO}_{n}$ on the space $\mathrm{Sym}_{n}$ of symmetric matrices. If $n$ is even then the nullcone $N_{\mathrm{Sym}_{n}^{\oplus k}}$ has two irreducible components for all $k \geq 1$. They are permuted by the elements of $O_{n} \backslash \mathrm{SO}_{n}$. If $n$ is odd, then $N_{\mathrm{Sym}_{n}^{\oplus k}}$ is irreducible for all $k \geq 1$.

Proof. For $n=2 \ell$, the positive roots of $\mathfrak{s o}_{2 \ell}$ are given by

$$
\Delta_{K}^{+}:=\left\{\varepsilon_{i} \pm \varepsilon_{j} \mid 1 \leq i<j \leq \ell\right\}
$$

and the weights of $V$ by

$$
X_{V}=\left\{ \pm\left(\varepsilon_{i}+\varepsilon_{j}\right) \mid 1 \leq i \leq j \leq \ell\right\} \cup\left\{ \pm\left(\varepsilon_{i}-\varepsilon_{j}\right) \mid 1 \leq i<j \leq \ell\right\} \cup\{0\},
$$

where the zero weight space has dimension $\ell-1$. The dimension of $V$ is $\left(\begin{array}{l}n \\ 2\end{array}\right)-1=$ $2 \ell^{2}+(\ell-1)$. Since the representation is self-dual every maximal unstable subset $X \subset X_{V}$ has $\ell^{2}$ elements. It is not difficult to see that an unstable $\Delta^{+}$-invariant subset $X$ is a subset of

$$
\left.\left\{\varepsilon_{i} \pm \varepsilon_{j}\right) \mid 1 \leq i<j \leq \ell\right\} \cup\left\{2 \varepsilon_{1}, 2 \varepsilon_{2}, \ldots, 2 \varepsilon_{\ell},-2 \varepsilon_{\ell}\right\} .
$$


It follows that there are exactly two nonequivalent maximal unstable $\Delta^{+}$-invariant subsets,

$$
\begin{aligned}
& X_{1}:=\left\{\varepsilon_{i} \pm \varepsilon_{j} \mid 1 \leq i<j \leq \ell\right\} \cup\left\{2 \varepsilon_{1}, 2 \varepsilon_{2}, \ldots, 2 \varepsilon_{\ell-1}, 2 \varepsilon_{\ell}\right\} \quad \text { and } \\
& X_{2}:=\left\{\varepsilon_{i} \pm \varepsilon_{j} \mid 1 \leq i<j \leq \ell\right\} \cup\left\{2 \varepsilon_{1}, 2 \varepsilon_{2}, \ldots, 2 \varepsilon_{\ell-1},-2 \varepsilon_{\ell}\right\} .
\end{aligned}
$$

It is well known that there is an element $g \in O_{2 \ell}$, stabilizing the given torus $T_{0}$, such that for the action on the roots we have $g \varepsilon_{i}=\varepsilon_{i}$ for $i<\ell$ and $g \varepsilon_{\ell}=-\varepsilon_{\ell}$. Hence, $g X_{1}=X_{2}$ and so $N_{V^{\oplus k}}=\mathrm{SO}_{n} V_{X_{1}}^{\oplus k} \cup \mathrm{SO}_{n} V_{X_{2}}^{\oplus k}$ has two irreducible components for any number $k$ of copies of $V$.

For $n=2 \ell+1$ the positive roots of $\mathfrak{s o}_{2 \ell+1}$ are given by

$$
\Delta_{K}^{+}:=\left\{\varepsilon_{i} \pm \varepsilon_{j} \mid 1 \leq i<j \leq \ell\right\} \cup\left\{\varepsilon_{i} \mid 1 \leq i \leq \ell\right\}
$$

and the weights $X_{V}$ of $V$ by

$$
\left\{ \pm\left(\varepsilon_{i}+\varepsilon_{j}\right) \mid 1 \leq i \leq j \leq \ell\right\} \cup\left\{ \pm\left(\varepsilon_{i}-\varepsilon_{j}\right) \mid 1 \leq i<j \leq \ell\right\} \cup\left\{ \pm \varepsilon_{i} \mid 1 \leq i \leq \ell\right\} \cup\{0\},
$$

where the zero weight space has dimension $\ell$. There is exactly one maximal unstable $\Delta^{+}$-invariant subset of $X_{V}$, namely

$$
X:=\left\{\varepsilon_{i}+\varepsilon_{j} \mid 1 \leq i \leq j \leq \ell\right\} \cup\left\{\varepsilon_{i}-\varepsilon_{j} \mid 1 \leq i<j \leq \ell\right\} \cup\left\{\varepsilon_{i} \mid 1 \leq i \leq \ell\right\} .
$$

Hence, the nullcone $N_{V^{\oplus k}}$ is irreducible for all $k \geq 1$.

Example 3.16 (Pairs of quadratic forms (the case $C_{m}$ )). Let $U$ be a vector space of dimension $m$ and consider $W:=U \oplus U^{*}$ with the nondegenerate skew form $\beta\left((u, \zeta),\left(u^{\prime}, \zeta^{\prime}\right)\right):=\zeta^{\prime}(u)-\zeta\left(u^{\prime}\right)$. Then $\operatorname{GL}(U)$ is naturally embedded into the corresponding symplectic group $G:=\operatorname{Sp}(W, \beta)$ by $\phi \mapsto\left(\phi,\left(\phi^{*}\right)^{-1}\right)$. Setting $\theta:=\operatorname{Int}\left(\operatorname{id}_{U},-\operatorname{id}_{U^{*}}\right)$, we find that $K:=G^{\theta}=\operatorname{GL}(U)$ and $V:=\left(\mathfrak{s p}(W, \beta)_{-1}=\right.$ $S^{2}(U) \oplus S^{2}\left(U^{*}\right)$. Thus $\operatorname{rk} G=\operatorname{rk} K$, and $V$ contains a Cartan subalgebra a, namely

$$
\mathfrak{a}:=\left\{\left(\sum_{i} a_{i} u_{i}^{2}, \sum_{i} a_{i}\left(u_{i}^{*}\right)^{2}\right) \mid\left(a_{1}, a_{2}, \ldots, a_{m}\right) \in \mathbb{C}^{m}\right\},
$$

where $\left(u_{1}, \ldots, u_{m}\right)$ is a basis of $U$ and $\left(u_{1}^{*}, \ldots, u_{m}^{*}\right)$ the dual basis of $U^{*}$. It follows that there are $2^{m}=\left[W_{\mathrm{Sp}(2 m)}: W_{\mathrm{GL}(m)}\right]$ maximal unstable subsets containing a given Borel subalgebra $\mathfrak{b}_{0}$ of $\mathfrak{g l}(U)$.

In order to describe $\mathscr{B}_{V}$ we use a basis of $U$ and its dual to obtain the usual identification $\mathfrak{s p}(W, \beta)=\mathfrak{s p}(2 m) \subset \mathfrak{g l}(2 m)$. Then the upper triangular matrices in $\mathfrak{s p}(2 m)$ form a Borel algebra $\mathfrak{b}$. The corresponding positive roots are

$$
\Delta^{+}=\left\{\varepsilon_{i}-\varepsilon_{j} \mid 1 \leq i<j \leq m\right\} \cup\left\{2 \varepsilon_{k} \mid 1 \leq k \leq m\right\}
$$

and contain the positive roots $\Delta_{K}^{+}=\left\{\varepsilon_{i}-\varepsilon_{j} \mid 1 \leq i<j \leq m\right\}$ corresponding to the Borel subalgebra $\mathfrak{b}_{0}:=\mathfrak{b} \cap \mathfrak{g l}(U)$. We have to describe all systems of positive roots $P \subset \Delta:=\Delta^{+} \cup-\Delta^{+}$which contain $\Delta_{K}^{+}$. Clearly, it suffices to describe 
the corresponding $\rho_{P}:=\sum_{\alpha \in P} \alpha$, since $P=\left\{\alpha \in \Delta \mid\left(\rho_{P}, \alpha\right)>0\right\}$. We have $\rho_{\Delta^{+}}=\sum(m-i+1) \varepsilon_{i}$ and $\rho_{P}=w \rho_{\Delta^{+}}$for some $w \in W$. Hence, $\rho_{P}$ has the form $\sum a_{i} \varepsilon_{i}$ where $\left\{\left|a_{i}\right|\right\}=\{1,2, \ldots, m\}$.

We call such a $P$ (or the corresponding $\rho_{P}$ ) admissible if it contains $\Delta_{K}^{+}$. We have the following inductive description, starting with $\mathfrak{s p}(2)=\mathfrak{s l}(2)$ and $P=\left\{\varepsilon_{1}\right\}$ or $P=\left\{-\varepsilon_{1}\right\}$.

Proposition 3.17. (1) The admissible $\rho_{P}$ 's are of the form

$$
\rho_{P}=m \varepsilon_{1}+\rho_{Q^{\prime}} \quad \text { or } \quad \rho_{P}=\rho_{Q^{\prime \prime}}-m \varepsilon_{m}
$$

where $Q^{\prime} \subset \Delta^{\prime}:=\left\{ \pm\left(\varepsilon_{i}-\varepsilon_{j}\right) \mid 2 \leq i<j \leq m\right\} \cup\left\{ \pm 2 \varepsilon_{k} \mid 2 \leq k \leq m\right\}$ and $Q^{\prime \prime} \subset \Delta^{\prime \prime}:=\left\{ \pm\left(\varepsilon_{i}-\varepsilon_{j}\right) \mid 1 \leq i<j \leq m-1\right\} \cup\left\{ \pm 2 \varepsilon_{k} \mid 1 \leq k \leq m-1\right\}$ are both admissible.

(2) There are exactly two admissible $P$ 's such that all simple roots are in $P \cap X_{V}$. For $m=2 \ell$ these are given by

$$
\begin{aligned}
& \rho_{P}=m \varepsilon_{1}+(m-2) \varepsilon_{2}+\cdots+2 \varepsilon_{\ell-1}-\varepsilon_{\ell}-3 \varepsilon_{\ell+1}-\cdots-(m-1) \varepsilon_{m} \quad \text { or } \\
& \rho_{P}=(m-1) \varepsilon_{1}+(m-3) \varepsilon_{2}+\cdots+\varepsilon_{\ell-1}-2 \varepsilon_{\ell}-4 \varepsilon_{\ell+1}-\cdots-m \varepsilon_{m}, \\
& \text { and for } m=2 \ell-1 \text { by } \\
& \rho_{P}=m \varepsilon_{1}+(m-2) \varepsilon_{2}+\cdots+\varepsilon_{\ell-1}-2 \varepsilon_{\ell}-4 \varepsilon_{\ell+1}-\cdots-(m-1) \varepsilon_{m} \quad \text { or } \\
& \rho_{P}=(m-1) \varepsilon_{1}+(m-3) \varepsilon_{2}+\cdots+2 \varepsilon_{\ell-1}-1 \varepsilon_{\ell}-3 \varepsilon_{\ell+1}-\cdots-m \varepsilon_{m} .
\end{aligned}
$$

In particular, the nullcone $N_{V}$ has two irreducible components.

Proof. (1) It is easy to see that the given elements are indeed of the form $\rho_{P}$ for some set $P$ of positive roots, and, by induction, we also see that they are admissible. Since their number is $2^{m}$ we found them all.

(2) We have to describe those admissible $P$ 's where no simple root of $P$ is contained in $\Delta_{K}^{+}$. Since $\rho_{P}=\sum_{i} a_{i} \varepsilon_{i}$ where $\left\{\left|a_{i}\right|\right\}=\{1,2, \ldots, m\}$ the claim easily follows.

Again one shows that the group $\bar{K}=\bar{G}^{\theta}$ has two connected components, in accordance with the fact that $\bar{K}$ has a dense orbit in $N_{V}$ (see Remark 3.12).

Example 3.18 (Pairs of skew forms (the case $D_{m}$ )). Proceeding in the same way as in the previous example, this time using the nondegenerate quadratic form $q(u, \zeta):=\zeta(u)$ on $W:=U \oplus U^{*}$ we obtain $G:=\operatorname{SO}(W, q), K:=G^{\theta}=\mathrm{GL}(U)$ and $V:=\bigwedge^{2} U \oplus \bigwedge^{2} U^{*}$. Again, $\operatorname{rk} G=$ rk $K$, but here $V$ does not contain a Cartan subalgebra of $\mathfrak{s o}(W, q)$. The number of admissible sets $P$ of positive roots equals $2^{m-1}$, but it is not clear which of them contribute to an irreducible component of the nullcone $N_{V}$. 


\section{The example of the 4-qubits}

The group $G:=\mathrm{SO}_{8}$ has an involution $\theta$ such that $K:=G^{\theta}=\mathrm{SO}_{4} \times \mathrm{SO}_{4}$ and $V=$ $M_{4}$ with the obvious action of $K$. This representation can be identified with the 4qubits, i.e., the representation of $\mathrm{SL}_{2} \times \mathrm{SL}_{2} \times \mathrm{SL}_{2} \times \mathrm{SL}_{2}$ on $\mathbb{C}^{2} \otimes \mathbb{C}^{2} \otimes \mathbb{C}^{2} \otimes \mathbb{C}^{2}$. The positive roots of $\mathfrak{k}=\mathfrak{s o}_{4} \oplus \mathfrak{s o}_{4}$ are given by $\Delta_{K}^{+}=\left\{\varepsilon_{1}-\varepsilon_{2}, \varepsilon_{1}+\varepsilon_{2}, \varepsilon_{3}-\varepsilon_{4}, \varepsilon_{3}+\varepsilon_{4}\right\}$ and the weights of $V$ by

$$
X_{V}=\left\{ \pm\left(\varepsilon_{1} \pm \varepsilon_{3}\right), \pm\left(\varepsilon_{1} \pm \varepsilon_{4}\right), \pm\left(\varepsilon_{2} \pm \varepsilon_{3}\right), \pm\left(\varepsilon_{2} \pm \varepsilon_{4}\right)\right\} .
$$

For a Borel subalgebra $\mathfrak{b}$ of $\mathfrak{s o}_{8}$ the half sums of positive roots $\rho_{\mathfrak{b}}$ has the form $\sum_{i} a_{i} \varepsilon_{i}$ where $\left\{\left|a_{1}\right|,\left|a_{2}\right|,\left|a_{3}\right|,\left|a_{4}\right|\right\}=\{0,1,2,3\}$. Moreover, $\mathfrak{b}$ contains the Borel subalgebra $\mathfrak{b}_{0} \subset \mathfrak{k}$ corresponding to $\Delta_{K}^{+}$if and only if $a_{1} \geq \pm a_{2}$ and $a_{3} \geq \pm a_{4}$. This gives the following $12\left(=\left[W: W_{K}\right]\right)$ weight vectors $\rho_{P}$ corresponding to the maximal unstable subspaces $V_{\mathfrak{b}} \in \mathscr{B}_{V}$ :

$$
\begin{array}{lll}
3 \varepsilon_{1}+2 \varepsilon_{2}+\varepsilon_{3}, & 3 \varepsilon_{1}+\varepsilon_{2}+2 \varepsilon_{3}, & 3 \varepsilon_{1}+2 \varepsilon_{3}+\varepsilon_{4}, \\
3 \varepsilon_{1}-2 \varepsilon_{2}+\varepsilon_{3}, & 3 \varepsilon_{1}-\varepsilon_{2}+2 \varepsilon_{3}, & 3 \varepsilon_{1}+2 \varepsilon_{3}-\varepsilon_{4}, \\
\varepsilon_{1}+3 \varepsilon_{3}+2 \varepsilon_{4}, & 2 \varepsilon_{1}+3 \varepsilon_{3}+\varepsilon_{4}, & 2 \varepsilon_{1}+\varepsilon_{2}+3 \varepsilon_{3}, \\
\varepsilon_{1}+3 \varepsilon_{3}-2 \varepsilon_{4}, & 2 \varepsilon_{1}+3 \varepsilon_{3}-\varepsilon_{4}, & 2 \varepsilon_{1}-\varepsilon_{2}+3 \varepsilon_{3} .
\end{array}
$$

Given a $\rho_{\mathfrak{b}}$ from the list we know that $K^{0} V_{\mathfrak{b}}$ is an irreducible component of $N_{V}$ if and only if $V_{\mathfrak{b}}$ contains the simple roots, i.e., if and only if $\mathfrak{b}_{0}$ does not contain a simple root with respect to $\mathfrak{b}$. If $\rho$ is of the form $\sum_{i} a_{i} \varepsilon_{i}$ this means that $a_{1} \pm a_{2} \geq 2$ and $a_{3} \pm a_{4} \geq 2$ which has the following four solutions:

$$
O_{0}:=\left\{3 \varepsilon_{1}+\varepsilon_{2}+2 \varepsilon_{3}, 3 \varepsilon_{1}-\varepsilon_{2}+2 \varepsilon_{3}, 2 \varepsilon_{1}+3 \varepsilon_{3}+\varepsilon_{4}, 2 \varepsilon_{1}+3 \varepsilon_{3}-\varepsilon_{4}\right\} .
$$

The group $S_{4}$ acts on the 4-qubits permuting the 4 factors in $\mathbb{C}^{2} \otimes \mathbb{C}^{2} \otimes \mathbb{C}^{2} \otimes \mathbb{C}^{2}$. The corresponding action on $\left(\mathrm{SL}_{2}\right)^{4}$ permutes the positive (simple) roots $\Delta_{K}^{+}$. It follows that $O_{0}$ is a single orbit under $S_{4}$ which implies that $S_{4}$ permutes the four irreducible components of $N_{V}$ transitively. The remaining $\rho$ 's from the list above decompose into 2 further orbits, namely

$$
\begin{aligned}
& O_{1}:=\left\{3 \varepsilon_{1}+2 \varepsilon_{3}+\varepsilon_{4}, 3 \varepsilon_{1}+2 \varepsilon_{3}-\varepsilon_{4}, 2 \varepsilon_{1}+\varepsilon_{2}+3 \varepsilon_{3}, 2 \varepsilon_{1}-\varepsilon_{2}+3 \varepsilon_{3}\right\} \text { and } \\
& O_{2}:=\left\{3 \varepsilon_{1}+2 \varepsilon_{2}+\varepsilon_{3}, 3 \varepsilon_{1}-2 \varepsilon_{2}+\varepsilon_{3}, \varepsilon_{1}+3 \varepsilon_{3}+2 \varepsilon_{4}, \varepsilon_{1}+3 \varepsilon_{3}-2 \varepsilon_{4}\right\} .
\end{aligned}
$$

The stabilizer of any one of the $\rho$ 's is $S_{3} \subset S_{4}$. Since $S_{3}$ is not contained in a proper subgroup of $S_{4}$ it follows that the orbits $O_{i}$ generate either none or one or four irreducible components of $N_{V^{k}}$ for a given $k>1$. In fact, we have:

Proposition 4.1. For $k \geq 2$ the nullcone $N_{Q_{4}^{\oplus k}}$ has 12 irreducible components, decomposing into 3 orbits of 4 elements under $S_{4}$. The dimensions are $8 k+4$, $8 k+3$ and $8 k+1$. 
The proof needs a little preparation. We first have to translate the setting above into the standard coordinates of $Q_{4}=\mathbb{C}^{2} \otimes \mathbb{C}^{2} \otimes \mathbb{C}^{2} \otimes \mathbb{C}^{2}$. We write $\mathbb{C}^{2}=\mathbb{C} e_{0} \oplus \mathbb{C} e_{1}$. Then the elements $e_{i j k \ell}:=e_{i} \otimes e_{j} \otimes e_{k} \otimes e_{\ell}(i, j, k, \ell \in\{0,1\})$ form a basis of $Q_{4}$. The corresponding weights in $K=\left(\mathrm{SL}_{2}\right)^{4}$ will be denoted by $\varepsilon_{i j k \ell}$. Note that $-\varepsilon_{i j k \ell}=\varepsilon_{i^{\prime} j^{\prime} k^{\prime} \ell^{\prime}}$ where $i+i^{\prime}=j+j^{\prime}=k+k^{\prime}=\ell+\ell^{\prime}=1$. The nondegenerate invariant form is induced by the standard symplectic form on $\mathbb{C}^{2}$, hence given by

$$
\left(e_{i j k \ell}, e_{i^{\prime} j^{\prime} k^{\prime} \ell^{\prime}}\right):=\left(i, i^{\prime}\right) \cdot\left(j, j^{\prime}\right) \cdot\left(k, k^{\prime}\right) \cdot\left(\ell, \ell^{\prime}\right)
$$

where $(0,0)=(1,1)=0$ and $(0,1)=-(1,0)=1$. Such forms exist on every $Q_{k}$ and are symmetric for even $k$ and skew-symmetric for odd $k$. The group $S_{4}$ acts on $Q_{4}$ by permuting the indices, hence normalizing the action of $K$ and leaving the form ( , ) invariant.

The positive roots of $\mathfrak{k}=\mathfrak{s l}_{2} \oplus \mathfrak{s l}_{2} \oplus \mathfrak{s l}_{2} \oplus \mathfrak{s l}_{2}$ are given by

$$
\Delta_{K}^{+}=\left\{\alpha_{1}:=\varepsilon_{1}+\varepsilon_{2}, \alpha_{2}:=\varepsilon_{1}-\varepsilon_{2}, \alpha_{3}:=\varepsilon_{3}+\varepsilon_{4}, \alpha_{4}:=\varepsilon_{3}-\varepsilon_{4}\right\},
$$

and we get

$$
\varepsilon_{i j k \ell}=\frac{1}{2}\left((-1)^{i} \alpha_{1}+(-1)^{j} \alpha_{2}+(-1)^{k} \alpha_{3}+(-1)^{\ell} \alpha_{4}\right) .
$$

For $\rho_{0}:=3 \varepsilon_{1}+\varepsilon_{2}+2 \varepsilon_{3}=2 \alpha_{1}+\alpha_{2}+\alpha_{3}+\alpha_{4} \in O_{0}$ the corresponding weight space $V_{0} \subset Q_{4}$ is given by

$$
V_{0}=\left\langle e_{0000}, e_{0011}, e_{0100}, e_{0001}, e_{0010}, e_{0101}, e_{0110}, e_{1000}\right\rangle=\left(e_{0} \otimes U\right) \oplus \mathbb{C} e_{1000},
$$

where

$$
U:=\left\langle e_{000}, e_{011}, e_{100}, e_{001}, e_{010}, e_{101}, e_{110}\right\rangle=\left(\mathbb{C} e_{000}\right)^{\perp} .
$$

Similarly, we find for $\rho_{1}:=3 \varepsilon_{1}+2 \varepsilon_{3}+\varepsilon_{4} \in O_{1}$ and $\rho_{2}:=3 \varepsilon_{1}+2 \varepsilon_{2}+\varepsilon_{3}$ the following weight spaces:

$$
\begin{aligned}
V_{1} & :=\left\langle e_{0000}, e_{0011}, e_{0100}, e_{0001}, e_{0010}, e_{0101}, e_{1000}, e_{1001}\right\rangle \\
& =\left\langle e_{000}, e_{001}, e_{010}, e_{100}\right\rangle \otimes \mathbb{C}^{2}, \\
V_{2} & :=\left\langle e_{0000}, e_{0001}, e_{0010}, e_{0100}, e_{0011}, e_{0101}, e_{0110}, e_{0111}\right\rangle \\
& =e_{0} \otimes Q_{3} .
\end{aligned}
$$

The normalizers of these spaces are $P_{0}:=B \times B \times B \times B, P_{1}:=B \times B \times B \times \mathrm{SL}_{2}$ and $P_{2}:=B \times \mathrm{SL}_{2} \times \mathrm{SL}_{2} \times \mathrm{SL}_{2}$.

\section{Lemma 4.2. The morphisms}

$$
K \times{ }_{P_{0}} V_{0} \rightarrow Q_{4}, \quad K \times_{P_{1}} V_{1} \rightarrow Q_{4}, \quad K \times_{P_{2}} V_{2} \rightarrow Q_{4}
$$

have finite degree. In particular,

$$
\operatorname{dim} K V_{0}^{\oplus k}=8 k+4, \quad \operatorname{dim} K V_{1}^{\oplus k}=8 k+3, \quad \operatorname{dim} K V_{2}^{\oplus k}=8 k+1 .
$$


Proof. Let $U_{-}:=\left\{\left[\begin{array}{ll}1 & 1 \\ u & 1\end{array}\right] \mid u \in \mathbb{C}\right\} \subset \mathrm{SL}_{2}$. It suffices to check that the morphisms

$$
\left(U_{-} \times U_{-} \times U_{-} \times U_{-}\right) \times V_{0} \rightarrow Q_{4}, \quad\left(U_{-} \times U_{-} \times U_{-}\right) \times V_{1} \rightarrow Q_{4}, \quad U_{-} \times V_{2} \rightarrow Q_{4}
$$

have rank $12=8+4,11=8+3$ and $9=8+1$, respectively, in a generic point of $e \in V_{0}, V_{1}$ and $V_{2}$; equivalently, that

$$
\begin{aligned}
\operatorname{dim}\left(\mathfrak{u}_{-} \oplus \mathfrak{u}_{-} \oplus \mathfrak{u}_{-} \oplus \mathfrak{u}_{-}\right) \cdot e+V_{0} & =12, \\
\operatorname{dim}\left(\mathfrak{u}_{-} \oplus \mathfrak{u}_{-} \oplus \mathfrak{u}_{-}\right) \cdot e+V_{1} & =11, \\
\operatorname{dim} \mathfrak{u}_{-} \cdot e+V_{2} & =9 .
\end{aligned}
$$

This is easy and left to the reader.

Lemma 4.3. Let $W \subsetneq Q_{3} a B \times B \times B$-stable subspace. Then a generic 2dimensional subspace of $Q_{3}$ is not conjugate to a subspace of $W$.

Proof. $\wedge^{2} W$ is stable under $B \times B \times B$ and $\operatorname{dim} \bigwedge^{2} W \leq\left(\begin{array}{l}7 \\ 2\end{array}\right)=21$. Therefore $\operatorname{dim}\left(\mathrm{SL}_{2} \times \mathrm{SL}_{2} \times \mathrm{SL}_{2}\right) \wedge^{2} W \leq 21+3<\left(\begin{array}{c}8 \\ 2\end{array}\right)=\operatorname{dim} \wedge^{2} Q_{3}$.

Proof of Proposition 4.1. According to Theorem 1.7 (and using Lemma 4.3) we have to show the following:

(a) There is a 2-dimensional subspace of $V_{2}$ which has no conjugate in either of the spaces $\sigma V_{0}$ or $\sigma V_{1}$ for $\sigma \in S_{4}$.

(b) There is a 2-dimensional subspace of $V_{1}$ which has no conjugate in either of the spaces $\sigma V_{0}$ for $\sigma \in S_{4}$.

Proof of (a). Some conjugate of a 2-dimensional subspace of $V_{0}$ has the form $e \otimes U$, where $e$ is an element of $\mathbb{C}^{2}$ and $U$ is an arbitrary 2-dimensional subspace of $Q_{3}$. It is easy to see that a subspace of $\sigma V_{0}$ or $\sigma V_{1}$ of this form is contained in $e \otimes W$, where $W$ is strictly contained in $Q_{3}$. Now the claim follows from Lemma 4.3.

Proof of (b). Let $U:=w \otimes \mathbb{C}^{2} \subset V_{1}$, where $w$ is a generic element in the subspace $\left\langle e_{000}, e_{001}, e_{010}, e_{100}\right\rangle$. Since $V_{0}$ has the form

$$
V_{0}=\left\langle e_{000}, e_{001}, e_{010}\right\rangle \otimes \mathbb{C}^{2} \oplus\left(\mathbb{C} e_{0110} \oplus \mathbb{C} e_{1000}\right)
$$

it follows that a conjugate of $U$ is contained in $V_{0}$ only if $w \in K^{\prime}\left\langle e_{000}, e_{001}, e_{010}\right\rangle$ where $K^{\prime}:=\left(\mathrm{SL}_{2}\right)^{3}$. But $K^{\prime}\left\langle e_{000}, e_{001}, e_{010}\right\rangle \subsetneq\left\langle e_{000}, e_{001}, e_{010, e_{100}}\right\rangle$ since we have $\operatorname{dim} K^{\prime}\left\langle e_{000}, e_{001}, e_{010}\right\rangle \leq 6$, because $\left\langle e_{000}, e_{001}, e_{010}\right\rangle$ is stable under a Borel of $K^{\prime}$, and $K^{\prime}\left\langle e_{000}, e_{001}, e_{010}, e_{100}\right\rangle$ is an irreducible component of the nullcone $N_{Q_{3}}$ of dimension 7 (see Example 2.4). 
The four images $\sigma V_{0}\left(\sigma \in S_{4}\right)$ have the following form (we use the transpositions $\sigma=(12),(13),(14))$ :

$$
\begin{aligned}
V_{0}^{\prime} & =\left\langle e_{000}, e_{001}, e_{100}\right\rangle \otimes \mathbb{C}^{2} \oplus\left(\mathbb{C} e_{1010} \oplus \mathbb{C} e_{0100}\right), \\
V_{0}^{\prime \prime} & =\left\langle e_{000}, e_{100}, e_{010}\right\rangle \otimes \mathbb{C}^{2} \oplus\left(\mathbb{C} e_{1100} \oplus \mathbb{C} e_{0010}\right), \\
V_{0}^{\prime \prime \prime} & =\left\langle e_{000}\right\rangle \otimes \mathbb{C}^{2} \oplus\left\langle e_{101}, e_{010}, e_{100}, e_{001}, e_{110}, e_{011}\right\rangle \otimes e_{0},
\end{aligned}
$$

and a similar argument applies.

\section{References}

[Borel and Mostow 1955] A. Borel and G. D. Mostow, "On semi-simple automorphisms of Lie algebras", Ann. of Math. (2) 61 (1955), 389-405. MR 16,897d Zbl 0066.02401

[Bürgin 2005] M. Bürgin, Nullforms, polarizations, and tensor powers, Dissertation, Universität Basel, 2005. in preparation.

[Derksen and Kemper 2002] H. Derksen and G. Kemper, Computational invariant theory, Encyclopaedia of Mathematical Sciences 130, Springer, Berlin, 2002. MR 2003g:13004 Zbl 1011.13003

[Derksen and Kraft 1995] H. Derksen and H. Kraft, "Constructive invariant theory", pp. 221-244 in Algèbre non commutative, groupes quantiques et invariants (Reims, 1995), Séminaires et Congrès 2, Soc. Math. France, Paris, 1995. Zbl 0883.13003

[Hilbert 1893] D. Hilbert, "Ueber die vollen Invariantensysteme", Math. Ann. 42:3 (1893), 313-373. MR 1510781 JFM 25.0173.01

[Joseph 1997] A. Joseph, "On a Harish-Chandra homomorphism”, C. R. Acad. Sci. Paris Sér. I Math. 324:7 (1997), 759-764. MR 98d:17017 Zbl 1002.17007

[Kempf 1978] G. R. Kempf, "Instability in invariant theory", Ann. of Math. (2) 108:2 (1978), 299316. MR 80c:20057 Zbl 0406.14031

[Kempken 1982] G. Kempken, Eine Darstellung des Köchers $\tilde{A}_{k}$, Bonner Mathematische Schriften 137, Universität Bonn, Mathematisches Institut, Bonn, 1982. MR 84a:14022 Zbl 0498.14021

[Kostant and Rallis 1971] B. Kostant and S. Rallis, "Orbits and representations associated with symmetric spaces”, Amer. J. Math. 93 (1971), 753-809. MR 47 \#399 Zbl 0224.22013

[Kraft 1984] H. Kraft, Geometrische Methoden in der Invariantentheorie, Aspects of Mathematics D1, Vieweg, Braunschweig, 1984. MR 86j:14006 Zbl 0669.14003

[Kraft et al. 1999] H. Kraft, L. W. Small, and N. R. Wallach, "Hereditary properties of direct summands of algebras", Math. Res. Lett. 6:3-4 (1999), 371-375. MR 2000g:16006 Zbl 0952.16005

[Luna and Richardson 1979] D. Luna and R. W. Richardson, "A generalization of the Chevalley restriction theorem”, Duke Math. J. 46:3 (1979), 487-496. MR 80k:14049 Zbl 0444.14010

[Mumford et al. 1994] D. Mumford, J. Fogarty, and F. Kirwan, Geometric invariant theory, 3rd ed., Ergebnisse der Mathematik (2) 34, Springer, Berlin, 1994. MR 95m:14012 Zbl 0797.14004

[Popov 2003] V. L. Popov, "The cone of Hilbert null forms", pp. 177-194 in Number theory, algebra, and algebraic geometry: papers dedicated to the 80th birthday of Igor Rostislavovich Shafarevich, Proceedings Steklov Inst. Math. 241, Amer. Math. Soc., Providence, 2003. MR 2005e:14071 Zbl 02174379

[Vinberg 1975] È. B. Vinberg, "On the linear groups associated to periodic automorphisms of semisimple algebraic groups”, Dokl. Akad. Nauk SSR 221 (1975), 767-770. In Russian; translated in Sov. Math. Dokl. 16 (1975), 406-409. MR 58 \#5954 Zbl 0334.20020 
[Vinberg 1976] È. B. Vinberg, "The Weyl group of a graded Lie algebra”, Izv. Akad. Nauk SSSR Ser. Mat. 40:3 (1976), 488-526, 709. In Russian; translated in Math. USSR Izv. 10 (1976), 463-495. MR 55 \#3175 Zbl 0371.20041

[Wallach 1993] N. R. Wallach, "Invariant differential operators on a reductive Lie algebra and Weyl group representations", J. Amer. Math. Soc. 6:4 (1993), 779-816. MR 94a:17014 Zbl 0804.22004

[Wallach 2005] N. R. Wallach, "The Hilbert series of measures of entanglement for 4 qubits", Acta Appl. Math. 86:1-2 (2005), 203-220. MR 2134319

Received February 21, 2005.

HANSPETER KRAFT

MATHEMATISCHES INSTITUT DER UNIVERSITÄT BASEL

RHEINSPRUNG 21

CH-4051 BASEL

SWITZERLAND

Hanspeter.Kraft@unibas.ch

NOLAN R. WALLACH

DEPARTMENT OF MATHEMATICS

University of CALIFornia, SAN Diego

9500 GILMAN DRIVE

LA JOLLA, CA 92093-0112

UNITED STATES

nwallach@ucsd.edu 